\title{
A Low Noise Receiver for Submillimeter Astronomy
}

Wengler, M., Woody, D., Miller, R., Phillips, T.

M. J. Wengler, D. P. Woody, R. E. Miller, T. G. Phillips, "A Low Noise Receiver for Submillimeter Astronomy," Proc. SPIE 0598, Instrumentation for Submillimeter Spectroscopy, (21 April 1986); doi: 10.1117/12.952315

SPIE Event: 1985 International Technical Symposium/Europe, 1985, Cannes, France 


\title{
A Low Noise Receiver for Submillimeter Astronomy
}

\author{
M. J. Wengler ${ }^{1}$, D. P. Woody ${ }^{1}$, R. E. Miller ${ }^{2}$ and T. G. Phillips \\ ${ }^{1}$ California Institute of Technology, Pasadena, California 91125 \\ ${ }^{2}$ AT\&T Bell Laboratories, Murray Hill. New Jersey 07974
}

\begin{abstract}
A broadband, low noise heterodyne receiver, suitable for astronomical use, has been built using a $\mathrm{Pb}$ alloy superconducting tunnel junction (SIS). The RF coupling is quasioptical via a bowtie antenna on a quartz lens and is accomplished without any tuning elements. In its preliminary version the double sideband receiver noise temperature rises from $205 \mathrm{~K}$ at $116 \mathrm{GHz}$ to $815 \mathrm{~K}$ at $466 \mathrm{GHz}$. This is the most sensitive broadband receiver yet reported for sub-mm wavelengths. Its multi-octave sensitivity and low local oscillator power requirements make this receiver ideal for remote ground observatories or space-borne telescopes such as NASA's Large Deployable Reflector. A version of this receiver is now being built for NASA's Kuiper Airborne Observatory.
\end{abstract}

\section{Introduction}

The theory of quantum mechanical mixing for quasiparticle tunnel junctions predicts that superconductor-insulator-superconductor junction (SIS) receivers can approach quantum limited sensitivity for $\mathrm{mm}$ and sub-mm wavelengths. ${ }^{1,2}$ The first reports of heterodyne mixing in SISs using quasiparticle effects indicated that they would make excellent low noise mm wave receivers. ${ }^{3,4}$ SISs in waveguide structures ${ }^{5,6,7,8,9}$ are among the most sensitive receivers in use for $\mathrm{mm}$ waves.

With the imminent operation of a number of sub-mm telescopes and with long range planning for space going telescopes such as NASA's Large Deployable Reflector (LDR), the importance of low noise heterodyne receivers for this band is increased. The exceptionally good performance of SIS receivers for $\mathrm{mm}$ wavelengths make them excellent candidates for a new sub-mm receiver technology.

Only this SIS receiver ${ }^{10}$ and one other ${ }^{5}$ have been operated at sub-mm wavelengths. Other sub-mm receivers in use include waveguide mounted Schottky diodes ${ }^{11,12,13}$ and corner-cube mounted Schottkys. ${ }^{14,15}$ The heterodyne InSb bolometer ${ }^{16}$ is the most sensitive receiver in the sub-mm and has been responsible for most of the interstellar medium spectroscopy in this band, but its very low instantaneous bandwidth $(\approx 1 \mathrm{MHz})$ limits its versatility.

The theory of SIS quasiparticle mixing ${ }^{2}$ can be used to predict excellent performance for frequencies as high as $4 \Delta / h$ where $\Delta$ is the superconducting energy gap of the material of the junction and $h$ is Planck's constant. For the junction used here that limit is over $1000 \mathrm{GHz}$. Reviews of many of the important aspects of SIS receivers for radio astronomy have been given by Phillips and Woody ${ }^{17}$ and by Tucker and Feldman. ${ }^{18}$

This paper describes the design, construction and performance of a quasioptical SIS receiver. Its performance is respectable for $\mathrm{mm}$ waves and is the best yet reported for any broadband sub-mm receiver. The design and performance of the mixer are presented, and its receiver performance is compared with other low-noise broadband systems.

\section{RF optics}

A major difficulty in receiver design at $\mathrm{mm}$ and sub-mm wavelengths is to provide efficient coupling of radiation to the detector over a reasonable spectral range. In the $\mathrm{mm}$ this is most effectively accomplished by placing the detector in a waveguide which has one or more tuning stubs. Radiation is coupled into the waveguide with feedhorns. In the sub-mm band waveguide dimensions become difficult to work with, for instance a $500 \mathrm{GHz}$ reduced height waveguide might have dimensions $.4 \mathrm{~mm}$ by .1 mm. In addition to the difficulties of making the detector and its circuitry small compared to these dimensions, surface roughness of the waveguide walls results in loss. Even at their best, waveguide structures seem limited to about a half octave of good performance.

An alternative to waveguide structures is the quasioptical corner reflector and long wire antenna, which is suitable for use with Schottky diodes ${ }^{14,15}$ because of the need for a whisker contact. These can have good performance over 3 octaves. To extend SIS receiver performance to higher frequencies, it is natural to use a photolithographic quasioptical structure. The mixer in this receiver uses a quasioptical coupling scheme which is similar to one which has been used for direct detection at $120 \mu \mathrm{m}$. ${ }^{19}$

The scheme consists of a bow-tie shaped planar antenna (bowtie) placed on the back of a quartz hyperhemisphere (a truncated sphere) with a plastic lens in front of it (Figs. 1 and 2). The features of this coupling scheme which are appealing to a mixer designer are best presented in the language of antenna design. They are: 1) the beam pattern of the antenna is $80 \%$ coupled to a forward lobe (and 20\% coupled backwards); 2) the antenna impedance is purely real; 3 ) the mixer and the antenna form a monolithic structure which minimizes parasitic reactances; 4 ) the beam pattern and impedance are independent of wavelength over a very large range; and 5) choking of the IF line to avoid RF propagation is achieved automatically by the bowtie antenna. These results are derived and experimentally verified in a review by Rutledge, Neikirk, and Kasilingam ${ }^{20}$ and a summary of that derivation is given below.

An infinitely long bowtie in vacuum is a lossless radial transmission line. This transmission line has some characteristic impedance $Z_{0}$ which is independent of frequency. The same transmission line in a dielectric would have a characteristic impedance $Z_{0} / n$ where $n$ is the index of refraction. If this transmission line is now placed on the planar boundary between a half-space of dielectric on the one side and vacuum on the other, it is no longer lossless. It becomes a very lossy transmission line of impedance $\sim Z_{0} / n_{m}$ where 


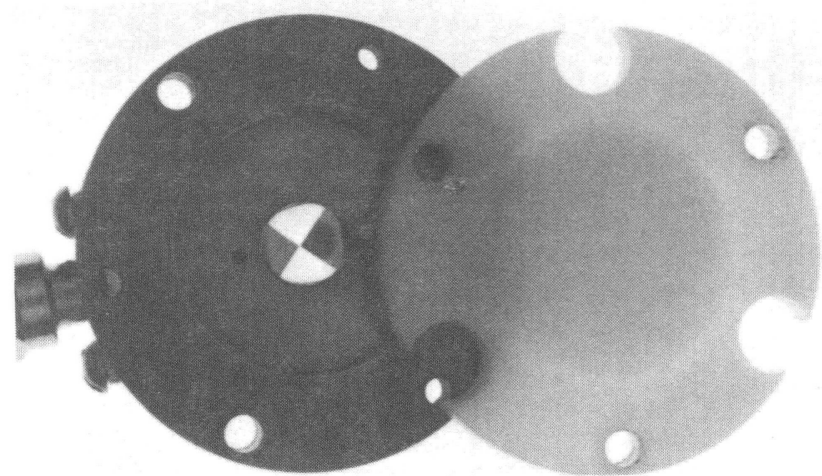

(a)

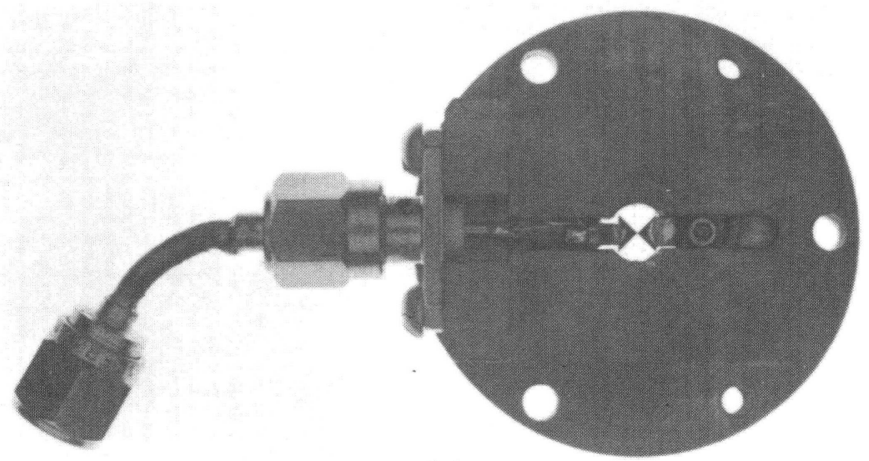

(b)

Figure 1. Photographs of the partially assembled mixer block; (a) From the RF side, the Teflon lens is shown out of place so that the bowtie can be seen through the fused quartz hyperhemisphere. (b) From the other side, the ground and IF connections to the bowtie can be seen. Spring contacts hold the SIS against the hyperhemisphere. The IF contact has $1.5 \mathrm{pF}$ capacitance to ground. The $70 \Omega$ co-ax shown acts as the IF matching transformer.

$n_{m}=\sqrt{\left(1+n^{2}\right) / 2}$. In traveling a distance $\lambda / n$ along the bowtie, a wave of free space wavelength $\lambda$ launches most of its energy into the dielectric, and a smaller amount into the vacuum. This energy forms an antenna beam pattern in the dielectric which is maximum perpendicular to the plane of the bowtie. A bowtie of length $2 L$ is an antenna with frequency independent properties for $\lambda<n L$. $\mathrm{RF}$ choking is not necessary for any IF connections made more than $\lambda / n$ from the center of the bowtie. For quartz, with $n=2$, the antenna beam pattern is $80 \%$ into the quartz.

The ideal bowtie has no upper frequency limit. The real bowtie will deviate from ideal performance if 1 ) the bowtie is not accurate on a scale of $\sim .1 \lambda$, or 2) the lenses become lossy, or 3) the conducting material of the bowtie becomes lossy. The SIS that detects the radiation is placed at the center of the bowtie and is shown in Fig. 3. Deviations from bowtie shape occur on the scale of microns which is much shorter than the wavelength at which loss in the fused quartz will be important $(\sim 100 \mu \mathrm{m})$. The electrical conductivity of the superconducting antenna metal is extremely high for frequencies up to $f \sim 2 \Delta / h$ which is about $500 \mathrm{GHz}$ for the lead alloys used here. As the frequency rises above this value, the lead alloy conductivity falls towards its normal state value. ${ }^{21}$ At what frequency this conductivity falls enough to affect performance is not yet known.

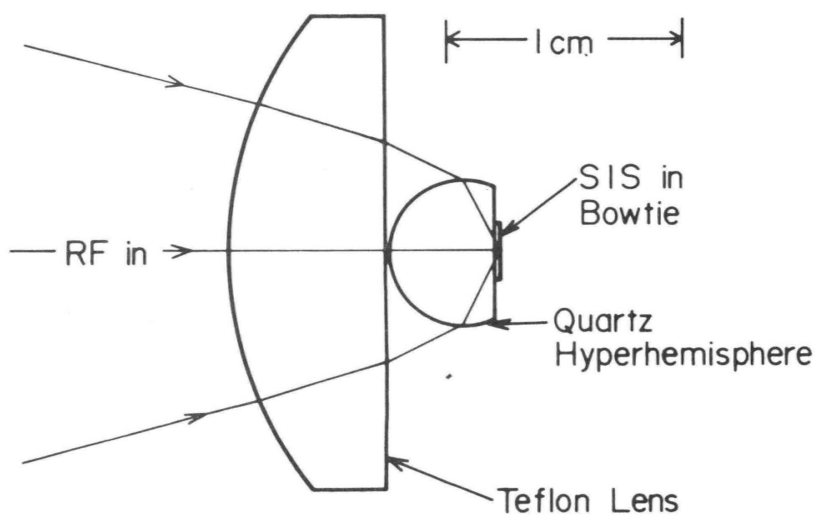

Figure 2. The RF coupling scheme. The outermost ray considered in the optics design is traced through the optics. The RF is incident as shown on a Teflon lens which, together with a quartz hyperhemisphere, focuses it into the center of a planar bowtie antenna which is integrated with an SIS.

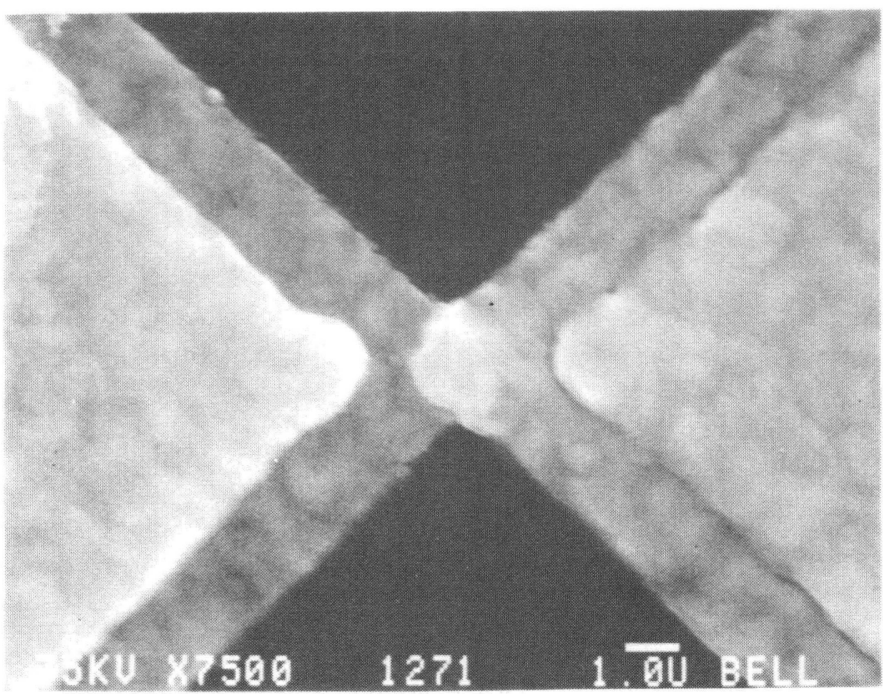

Figure 3. An SEM photograph of an SIS at the center of a bowtie. The $\sim 1 \mu \mathrm{m} \times 1 \mu \mathrm{m}$ overlap of the two metal films is the SIS. The bowtie pattern is seen to continue accurately down to a scale of a few microns.

The antenna impedance of a $90^{\circ}$ bowtie on dielectric with $n=2$ has been measured to be $120 \Omega^{20}$ and it has been suggested that this bowtie will have a beam pattern which is approximately symmetric in the $\mathbf{E}$ and $\mathbf{H}$ planes. ${ }^{22}$

In this mixer, the optics are designed to illuminate the bowtie pattern out to $f / .3$ in the quartz. The hyperhemisphere of quartz transforms this beam to $f / 1$ with no spherical aberration. ${ }^{20}$ The lens in front of the quartz can be chosen to give further focusing as desired. The plastic lens used in this work was designed to further transform the beam to $f / 1.9$. 


\section{Receiver configuration}

The principal parts of the receiver are the SIS detector, the bowtie optics, the mixer block, the IF amplifier chain, and the local oscillator (LO) chain.

The SIS detector used is essentially a diode and detects RF through rectification. A scanning electron microscope photograph of one is shown in Fig. 3. The devices reported here are made using a tri-level photoresist stencil technique. ${ }^{23,24}$ They differ from the $\mathrm{PbInAu}$ junctions described in Woody, Miller and Wengler ${ }^{8}$ only in the geometry of the films. The mask from which the junctions are made is fabricated by standard electron beam lithography. The pattern of the mask deviates from a bowtie in the central few microns where the .1 to $2(\mu \mathrm{m})^{2}$ overlap junctions are to be fabricated. Above the superconducting transition temperature these tunnel junctions have resistances $\left(R_{N}\right)$ of 20 to $200 \mathrm{ohms}$. The junction used in this experiment had $R_{N}=50 \Omega$. The integrated antenna and detector structures are fabricated on fused quartz substrates of dimensions $2.5 \mathrm{~mm}$ square by $.1 \mathrm{~mm}$ thick.

The optical elements are displayed schematically in Fig. 2 and photographically in Fig. 1. The fused quartz hyperhemisphere has a radius $r=3.3 \mathrm{~mm}$ and a length of $4.9 \mathrm{~mm}$. When assembled, the quartz substrate with the bowtie on it is placed on to the flat face of the hyperhemisphere. The lens placed in front of the quartz hyperhemisphere is plano-convex, machined from Teflon. It has a design focal length of $39.5 \mathrm{~mm}$.

The mixer block is made from OFHC copper and mechanically supports the lenses, the SIS and the IF circuitry. The mixer block has the job of illuminating the $20 \%$ of the bowtie beam pattern which is coupled out on the vacuum side of the antenna. This is terminated at low temperature in the mixer block by absorbing paint on all metal surfaces behind the plane of the bowtie. The block has built into it a superconducting coil capable of imposing a few hundred Gauss magnetic field onto the SIS detector. This allows suppression of Josephson or paired electron currents which cause hysteresis in the current-voltage $(I-V)$ characteristic of the SIS.

The IF circuit with the SIS is made by grounding one side of the bowtie, and connecting the other with a short wire to a coaxial connector. The IF is shunted to ground by a $1.5 \mathrm{pF}$ capacitor immediately where the wire joins the bowtie. This shorts out currents at frequencies above the desired IF passband, to help avoid saturation. ${ }^{7,25}$ Just beyond the coaxial connector is a short length of $70 \Omega$ co-ax which acts as a matching transformer for the IF band from 1.2 to $1.7 \mathrm{GHz}$.

The IF chain for these measurements had a coolable isolator ${ }^{26}$ before the first stage amplifier so that IF chain noise would be independent of input match, allowing the mixer noise to be measured. The first IF amplifier is a cooled GaAsFET 1.2-1.7 GHz amplifier based on the design of Weinreb, Fenstermacher, and Harris. ${ }^{27}$ This amplifier is cooled to liquid helium temperatures.

All the pieces mentioned so far are mounted on the work surface of a liquid helium dewar. The RF enters the dewar through a $.025 \mathrm{~mm}$ mylar vacuum window. It then passes through a $.7 \mathrm{~mm}$ thick quartz window and a thin sheet of black polyethylene mounted on a liquid nitrogen cooled radiation shield.

Optimum performance of an SIS mixer is achieved with an absorbed LO power $\sim(h f / e)^{2} / R_{N}$. This is $<.1 \mu \mathrm{W}$ at $500 \mathrm{GHz}$ for $R_{N}=50 \Omega$. Even allowing for a low coupling efficiency of LO power from the source to the mixer, only a few $\mu \mathrm{W}$ of power are required from the LO source. The fundamental LO used for these measurements was a klystron tuned to $116.5 \mathrm{GHz}$. The power was either used directly, or it was used to drive a GaAs Schottky diode frequency multiplier. ${ }^{28}$ The LO thus produced was coupled into the $\mathrm{RF}$ signal path by reflecting it from a mylar beam splitter of thickness $25 \mu \mathrm{m}$ for the lower 3 frequencies and $86 \mu \mathrm{m}$ for the $466 \mathrm{GHz}$ measurement. The calculated signal loss due to the beam splitter is <3\% up to $349 \mathrm{GHz}$, and $\sim 20 \%$ for $466 \mathrm{GHz}$.

\section{Receiver performance}

Receiver performance was measured both with and without magnetic field applied, except at $116.5 \mathrm{GHz}$. Ideally, application of magnetic field would cause suppression of Josephson effect currents and therefore the noise and $I-V$ curve hysteresis they cause. With the junctions used here, only partial alleviation of these effects was possible, and this was always accompanied by a small degradation of the non-linearity of the $I-V$ curve.

The primary performance measurement is of the response to hot $(290 \mathrm{~K})$ and cold $(80 \mathrm{~K})$ Eccosorb AN-72 loads placed in front of the LO injection beam splitter. The SIS is cooled to $\sim 4.2 \mathrm{~K}$ for these measurements. For each measurement there are two tuning parameters; the dc bias voltage and the LO power level. A range of bias voltages and LO powers are tried to see which gives the lowest receiver noise temperature, $T_{R}$. This method of measurement is intrinsically double sideband (DSB) so all results given are DSB. The lack of tuning structures and the wide RF bandwidth of the receiver imply that sideband response will be balanced.

The IF power is measured through a 1.3 to $1.5 \mathrm{GHz}$ bandpass filter. The linear portion of the SIS $I-V$ characteristic provides a reliable and calculable shot noise source which is used to calibrate the gain and noise $(19 \pm 1 \mathrm{~K})$ of the IF amplifier chain. ${ }^{8}$ These can be removed from the receiver measurements to give the gain and noise of the mixer.

Receiver performance was measured at four frequencies from 116.5 to $466 \mathrm{GHz}$. The best $T_{R}$ at each frequency is shown graphically in Fig. 4. The corresponding mixer gain and noise are also shown. No corrections have been made for any signal path losses or mismatches. For the lower frequencies the best results are achieved with no magnetic field applied to the junction. At $466 \mathrm{GHz}$ the mixer had IF power outputs which changed too quickly with dc bias voltage to allow reliable receiver performance calculations, except when magnetic field was applied. The raw data for the $466 \mathrm{GHz}$ measurement with field are shown in Fig. 5.

The mixer noise is proportional to LO frequency and is not affected significantly by the magnetic field. The mixer gain, however, is degraded by the addition of magnetic field, especially as frequency is increased. The dominant effect of the field is supposed to be the suppression of Josephson currents. If that is the case, then Josephson currents add to the conversion efficiency of the mixer as frequency is increased, and this is done without changing the mixer noise. 


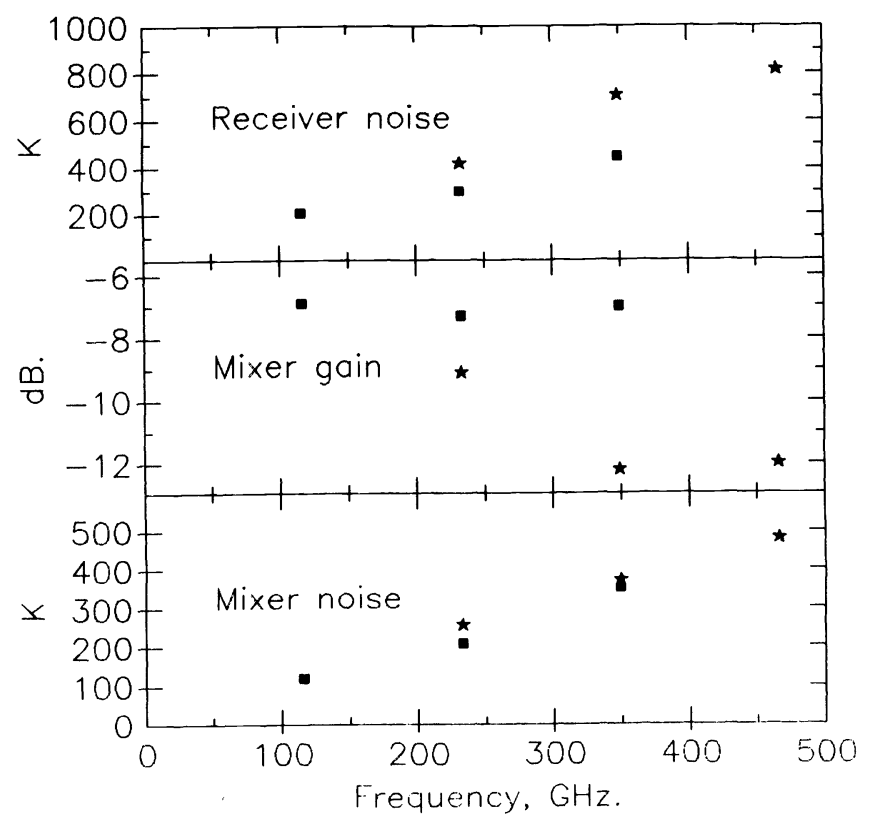

Figure 4. Mixer and receiver double sideband performance vs. LO frequency. $\star$ are measured with magnetic field applied to the junction and - are measured with no magnetic field. These are the values obtained when tuning for lowest $T_{R}$. No signal loss corrections have been made.

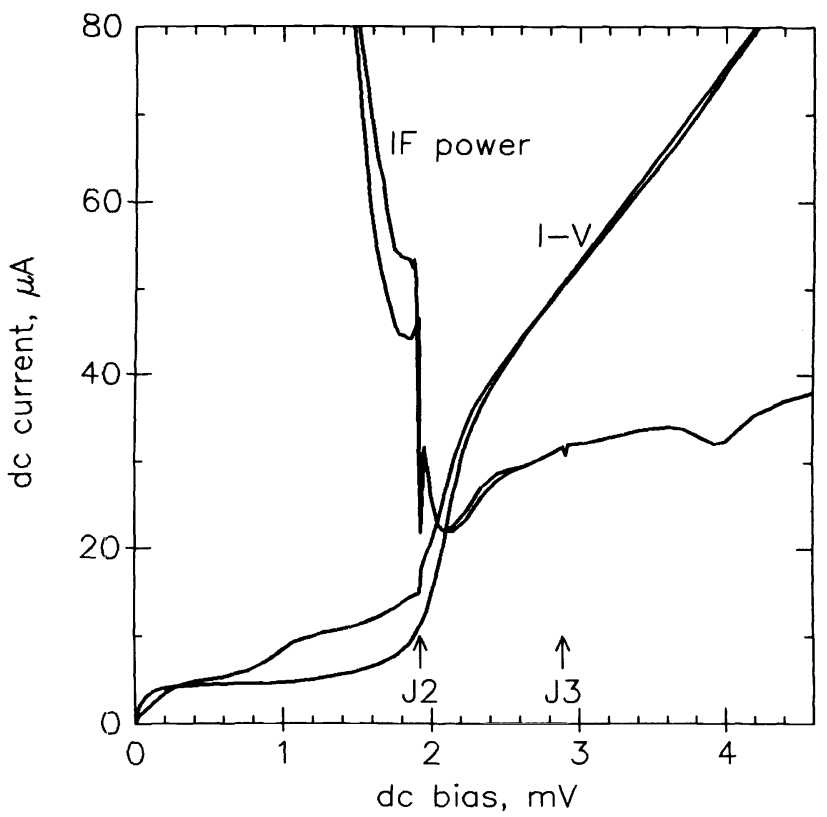

Figure 5. Receiver performance at $466 \mathrm{GHz}$ with magnetic field applied to the junction. The $I-V$ curves with and without LO applied are shown. IF power (arbitrary units) vs. dc bias voltage is shown for hot and cold loads held at the receiver input. The voltages marked J2 and J3 are where the ac Josephson frequency is respectively twice and three times the LO frequency.

In Fig. 6, the performance of this receiver is compared with the performance of the best reported waveguide mounted SIS ${ }^{5,6}$ and Schottky ${ }^{11,12,13}$ receivers, and with the best corner-reflector mounted Schottkys ${ }^{14}$. For receivers which report single sideband receiver temperatures, $T_{R}$ is taken to be half that value. $T_{R}$ is normalized to the quantum lower limit of $h \nu / 2 k_{B}$ for double sideband detection. It is seen in this comparison that SIS receivers are more sensitive than Schottky receivers to as high a frequency as the SISs have been measured. It is also seen that for each detector, the best receivers at lower frequencies are waveguide mounted while the best receivers at higher frequencies are quasioptically coupled.

The beam pattern of the mixer was roughly measured at 116.5 and $233 \mathrm{GHz}$. It was found to be the same at these two frequencies, with a FWHM of $14^{\circ} \pm 2^{\circ}$ in both $\mathbf{E}$ and $\mathbf{H}$ planes. The mixer was taken to the Owens Valley Radio Observatory (OVRO) and put in place of one of the $115 \mathrm{GHz}$ receivers on one of the $10.4 \mathrm{~m}$ dishes. At the receiver focus, a $15^{\circ}$ half width beam will fully illuminate the primary, so the bowtie beam would be expected to couple to the telescope somewhat inefficiently. Observations of the moon showed that $60 \%$ of the beam power making it out of the receiver dewar was making it to the moon, at both 115 and $230 \mathrm{GHz}$. Presumably, a shorter focal length plastic lens on the mixer would improve the coupling. Fig. 7 shows of the $\mathrm{J}=2-1$ and the $\mathrm{J}=1-0$ rotational transitions of carbon monoxide observed in the Orion molecular cloud. This verifies the astronomical usefulness of this receiver.

\section{Conclusions and future directions}

The bowtie on dielectric antenna structure integrated with an SIS junction has demonstrated good sensitivity over a bandwidth of two octaves with no RF tuning structures. Even in this initial experiment, receiver noise temperatures are the best reported for SIS or Schottky diode receivers at sub-mm wavelengths and it is expected $T_{R}$ can be lowered by cooling the SIS below $4.2 \mathrm{~K}$. Because of its frequency versatility without apparent loss of sensitivity, a mixer like this should be ideal for ground-based and space-borne sub-mm astronomy. As the weather at an observatory changes, so can the frequency of observation, allowing maximum efficiency of telescope time usage. In a space-borne system such as NASA's LDR this mixer will cover a band which would require four or more waveguide based mixers. The SIS's low LO power requirement yields savings in the weight, power and volume of this component of the receiver.

Another advantage of the bowtie design for astronomy is the relatively easy construction of an imaging array of bowties. The bowtie sits in a focal plane of the telescope, and with proper optical design many bowties can be placed in this focal plane to simultaneously sample many pixels on the sky. A waveguide based system would require a separate feedhorn and mixer for each pixel. In fact, two dimensional imaging arrays at $120 \mu \mathrm{m}$ using bowties in this fashion have already been built. ${ }^{19}$

The high frequency limit of SIS heterodyne receivers has not been measured in this experiment. Rather, good performance is shown to be possible to at least $466 \mathrm{GHz} \sim 1.8 \Delta / h$. Improvements in multiplier output and measurements with a molecular laser local oscillator will determine the high frequency limits of this device in the near future. If the Josephson effect is contributing substantially to mixer performance, it may be that $T_{R}$ will be satisfactory even above $4 \Delta / h \approx 1000 \mathrm{GHz}$.

Finally, in this receiver no advantage has been taken of the possibilities of micron scale circuitry to tune out the SIS capacitance at some frequency, as was done successfully in D'Addario's waveguide mixer. ${ }^{7}$ At this time, SISs in bowties are being fabricated with tuning circuitry to investigate this possibility. 


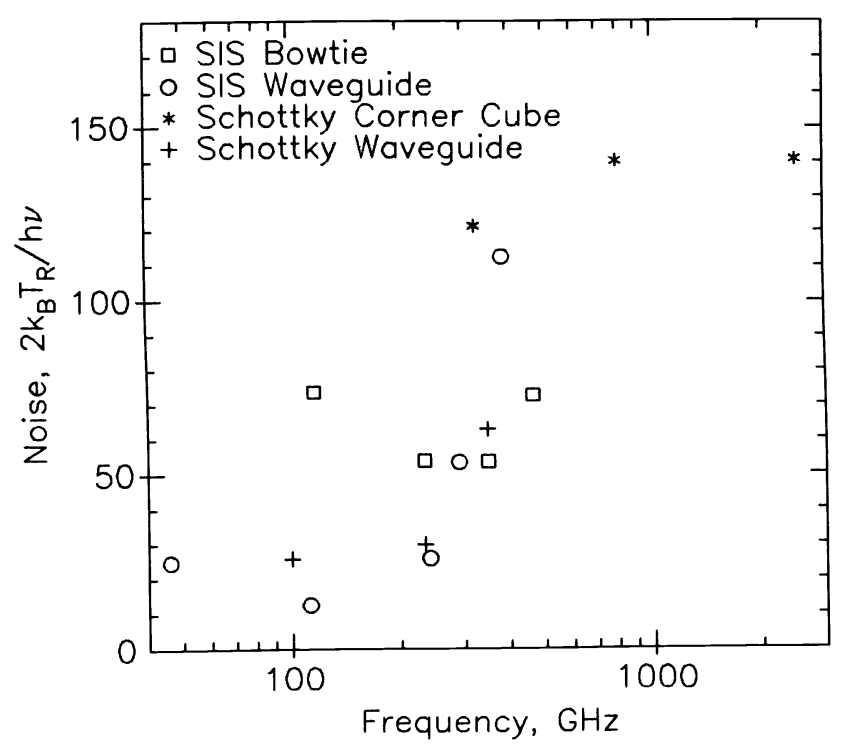

Figure 6. Comparison of $T_{R}$ of the best SIS and Schottky diode astronomical receivers reported in the literature. The best of waveguide and open structures are presented separately for comparison. References are given in the text.

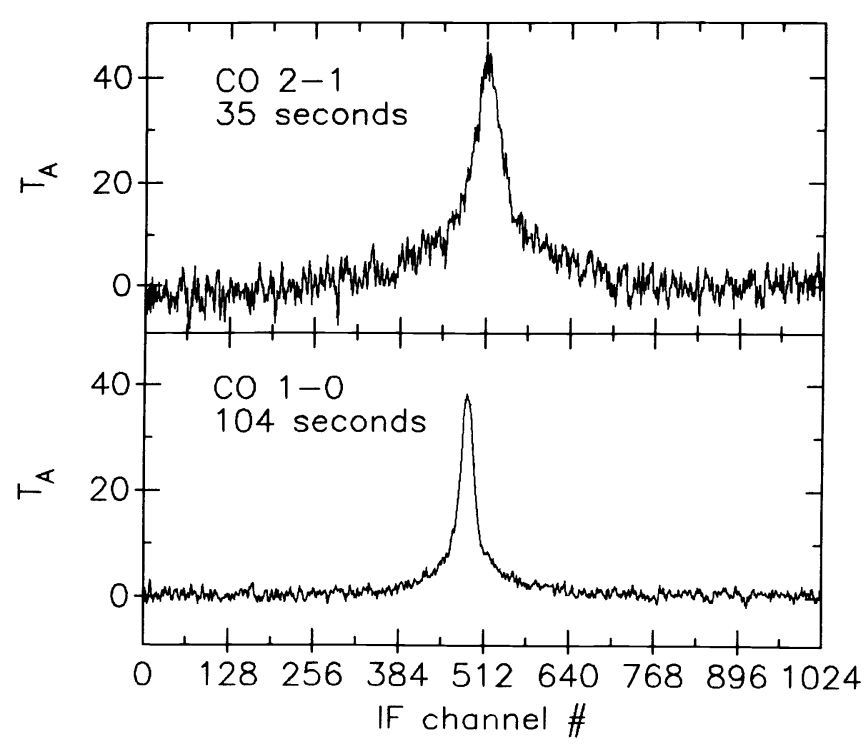

Figure 7. Astronomical observations of two $\mathrm{CO}$ transitions in Orion taken with this receiver at OVRO. The data are taken in 1024 channels spaced $.5 \mathrm{MHz}$ apart. The center channel corresponds to line frequencies of $230.531 \mathrm{GHz}$ for the $\mathrm{J}=2-1$ transition and $115.268 \mathrm{GHz}$ for $\mathrm{J}=1-0$. Signal was integrated for the times shown.

\section{Acknowledgements}

We are indebted to Dean Neikirk of the University of Texas and David Rutledge of Caltech for discussions about bowties which stimulated this project. At AT\&T Bell Labs we thank A. A. Stark for help with SIS mask construction and M. V. Schneider and P. Verlangieri for Schottky diodes used in the LO multipliers. At Caltech we are pleased to acknowledge G. A. Blake's help in the lab and at the Owens Valley Radio Observatory.

The work at Caltech was supported by NSF grants AST-8214693 and 8311849.

\section{References}

1. J. R. Tucker and M. F. Millea, "Photon detection in nonlinear tunneing devices," Appl. Phys. Lett., vol. 33, pp. 611-613, Oct. 1978

2. J. R. Tucker, "Quantum limited detection in tunnel junction mixers," IEEE J. Quantum Electron., vol. QE-15, pp. 1234-1258, Nov. 1979

3. P. L. Richards, T. M. Shen, R. E. Harris and F. L. Llyod, "Quasiparticle heterodyne mixing in SIS tunnel junctions," Appl. Phys. Lett., vol. 34, pp. 345-347, Mar. 1, 1979.

4. G. J. Dolan, T. G. Phillips and D. P. Woody, "Low-noise 115-GHz mixing in superconducting oxide-barrier tunnel junctions," Appl. Phys. Lett., vol. 34, pp. 347-349, Mar. 1, 1979.

5. E. C. Sutton, "A superconducting tunnel junction receiver for $230 \mathrm{GHz}$," IEEE Trans. Microwave Theory Tech., vol. MTT-31, pp. 589-592, July 1983.

6. S.-K. Pan, M. J. Feldman, A. R. Kerr, and P. Timbie, "Low-noise 115-GHz receiver using superconducting tunnel junctions," Appl. Phys. Lett., vol. 43, pp. 786-788, Oct. 1983.

7. Larry R. D'Addario, "An SIS Mixer for $90-120 \mathrm{GHz}$ with Gain and Wide Bandwidth," Intl. J. of IR and Millimeter Waves, vol. 5, pp. 1419-1442, 1985.

8. D. P. Woody, R. E. Miller, and M. J. Wengler, "85-115 GHz Receivers for Radio Astronomy," IEEE Trans. Microwave Theory Tech., vol. MTT-33, pp. 90-95, Feb. 1985.

9. K. H. Gundlach, R. Blundell, J. Ibruegger. E. J. Blum, "SIS quasiparticle mixer receiver for radio-astronomy applications," Proceedings of IC SQUID 1985, Berlin, June 1985.

10. M. J. Wengler, D. P. Woody, R. E. Miller and T. G. Phillips, "A low noise receiver for millimeter and submilimeter wavelengths," Intl. J. of IR and Millimeter Waves, vol. 6, pp. 697-706, Aug. 1985.

11. Neal R. Erickson, Digest of the Eighth Int'l Conference on IR and MM Waves, p. M6.7, 1983.

12. C. R. Predmore, A. V. Räisänen, N. R. Erickson, P. F. Goldsmith, and J. L. R. Marrero, "A broad-band, ultra-low-noise Schottky Diode Mixer Receiver from 80 to 115 GHz," IEEE Trans. Microwave Theory Tech., vol. MTT-32, pp. 498-506, May 1984.

13. Neal R. Erickson, "A very low noise single sideband receiver for 200-260 GHz," Preprint \#260 of Five College Radio Astronomy Observatory, 1985.

14. H. P. Röser, E. J. Durwen, R. Wattenbach, and G. V. Schultz, "Investigation of a heterodyne receiver with open structure mixer at $324 \mathrm{GHz}$ and $693 \mathrm{GHz}, "$ Intl. J. of IR and Millimeter Waves, vol. 5, pp. 301-314, March 1984.

Also: H. P. Röser, R. Wattenbach, and P. van der Wal, "Tunable heterodyne receiver from $100 \mu \mathrm{m}$ to $1000 \mu \mathrm{m}$ for airborne observations," Airborne Astronomy Symposium, NASA Conference Publication 2353, pp. 330-334, 1984.

15. A. Betz and J. Zmuidzinas, "A $150 \mu \mathrm{m}$ to $500 \mu \mathrm{m}$ heterodyne spectrometer for airborne astronomy," Airborne Astronomy Symposium, NASA Conference Publication 2353, pp. 320-329, 1984.

16. T. G. Phillips and K. B. Jefferts, "A low temperature bolometer heterodyne receiver for millimeter wave astronomy," Rev. Sci. 
Inst., vol. 44, pp. 1009-1014, Aug. 1973.

17. T. G. Phillips and D. P. Woody, "Millimeter- and submillimeter-wave receivers," Ann. Rev. Astron. Astrophys., vol. 20, pp. 285-321, 1982.

18. John R. Tucker and Marc J. Feldman, "Quantum Detection at Millimeter Wavelengths," Rev. Modern Phys., in press, 1985.

19. Dean P. Neikirk, Peter P. Tong, David B. Rutledge, Hyeon Park, and Peter E. Young, "Imaging antenna array at $119 \mu \mathrm{m}$," Appl. Phys. Lett., vol. 41, pp. 329-331, Aug. 1982.

20. D. B. Rutledge, D. P. Neikirk, and D. P. Kasilingam, "Integrated-Circuit Antennas" in Infrared and Millimeter Waves, K. J. Button, Ed., vol. 10, pp. 1-90, New York: Academic Press, 1984.

21. M. Tinkham, Introduction to Superconductivity, New York:McGraw-Hill, 1975.

22. D. B. Rutledge, private communication, Feb. 1984.

23. L. N. Dunkleberger, "Stencil technique for the preparation of thin-film Josephson devices," J. Vac. Sci. Tech., vol. 15, pp. 88-90, Jan. 1978

24. G. J. Dolan, "Offset masks for lift-off photoprocessing," Appl. Phys. Lett., vol. 31, pp. 337-339, Sept. 1977.

25. A. D. Smith and P. L. Richards, "Analytic solutions to superconductor-insulator-superconductor quantum mixer theory," $J$. Appl. Phys., vol. 53, pp. 3806-3812, May 1982.

26. Model LTE-1138 coolable isolator, Pamtech, Canoga Park, California, 91304, USA.

27. S. Weinreb, D. L. Fenstermacher, R. W. Harris, "Ultra-low-noise 1.2 to $1.7 \mathrm{GHz}$ cooled GaAsFET amplifiers," IEEE Trans. Microwave Theory Tech., vol. MTT-30, pp. 849-853, June 1982.

28. M. V. Schneider, "Metal-Semiconductor Junctions as Frequency Converters" in Infrared and Millimeter Waves, K. J. Button, Ed., vol. 6, pp. 210-275, New York: Academic Press, 1982. 